\title{
Electromechanical vibratory impact systems with kinetic energy losses
}

\author{
V.Yu. Neyman \\ Novosibirsk State Technical University \\ Novosibirsk, Russian Federation \\ e-mail: nv.nstu@ngs.ru
}

\author{
L.A Neyman \\ Novosibirsk State Technical University \\ Novosibirsk, Russian Federation \\ E-mail: neyman31@gmal.com
}

\begin{abstract}
The paper considers the processes of interaction between electrical, magnetic and mechanical parts of electromechanical vibratory impact systems. The impact energy exchange accompanied by kinetic energy transfer into deformed medium is analyzed. It is shown that this process is related to the energy loss when impact masses are deformed.

The characteristics reflecting the influence of design factors of the impact system with kinetic energy loss on deformation of impact masses have been obtained.
\end{abstract}

The expression describing kinetic energy transfer efficiency with respect to energy losses has been derived.

Keywords - electromagnetic vibratory impact system; linear electromagnetic drive; impact system; kinetic energy loss; interaction between impulses forces.

\section{INTRODUCTION}

Electromechanical vibratory systems with electromagnetic excitation are applied in different engineering areas for implementation of many technological processes in industry [1-4].

Development of mechanisms and machines on the base of electromagnetic vibratory drives are deeply considered in [5].

Synchronous electromagnetic impact machines wherein the striker motion frequency is multiple of the voltage pulses from power source are highly efficient for low-frequency impact technologies [6].

The existing procedures for calculation and design of electromagnetic machines are mostly based on static approaches. Hence it is difficult to use these procedures in the analysis and synthesis [7, 8]. Therefore it is necessary to execute the dynamic design with respect to many interrelated factors depending on power loss, motion velocity, frequency, and magnetic material nonlinear characteristics [9-16].

\section{STATEMENT OF PURPOSE}

Some variants of synchronous impact electromagnetic machines with short analysis of interaction between electrical, magnetic and mechanical systems are considered in [6]. Reciprocating motion of the impact mass is similar for many types of machines.

Fig. 1-6 show the variants of synchronous impact electromagnetic machines schemes and the respective diagrams of the striker travel $x$, the winding voltage $u$ and the current $i$.
The windings are powered by a $50 \mathrm{~Hz}$ single-phase source. In Fig. 2-6 $u_{\mathrm{tr}}, i_{\mathrm{tr}}$ stand for travel voltage and current, and $u_{\mathrm{rv}}, i_{\mathrm{rv}}$ stand for reverse ones.
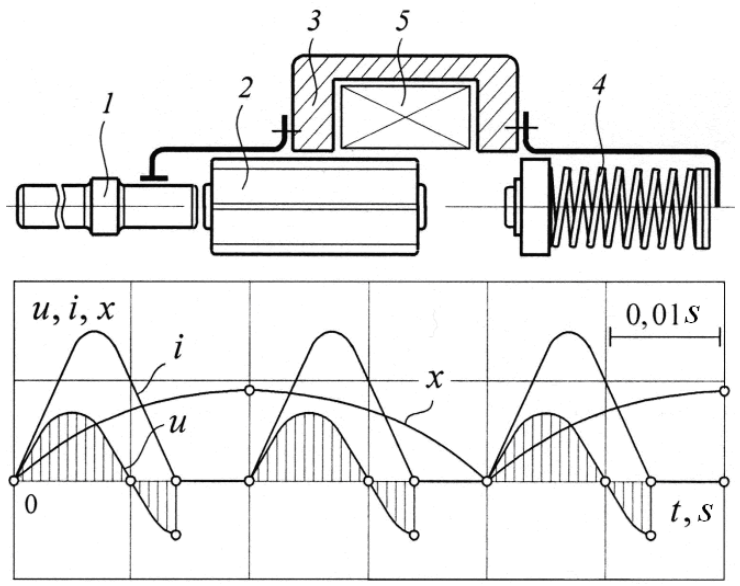

Fig. 1. Synchronous electromagnetic machine with a two-sided free runningout of the striker in the winding

The electromagnetic synchronous machine impact unit (Fig.1) combines the magnetic and mechanical elements: tool 1 , striker (forming the impact mass) -2 , magnetic core -3 , reverse spring buffer -4 , inertial converter heavier than striker combined in some cases with the reverse spring buffer -7 , magnetizing winding -5 (Fig. 1) or travel magnetizing winding -5 and reverse magnetizing winding in Fig. 2-6. The existing engineering solutions in such machines help to achieve the synchronous mode stability. Among single winding schemes the synchronous electromagnetic machine with a twosided free running-out of the striker has been brought into practice (Fig. 1).

The scheme of this machine was applied in handheld impact tools MC-15, MC-16.

The total operating cycle of the machine impact unit consists of voltage periods, providing synchronous impact frequency $n_{\mathrm{im}}=25 \mathrm{~s}^{-1}\left(1500 \mathrm{~min}^{-1}\right)$ and operating cycle duration $t_{\mathrm{c}}=0,04 \mathrm{~s}$.

The machine is powered by a single-phase source through a half-period rectifier. The striker travel towards the tool is 
executed by winding electromagnetic forces and the spring buffer elastic force. The reverse is made only by winding electromagnetic forces. Two-winding schemes are distinguished by wider capabilities of operating cycle implementation. Synchronous impact electromagnetic machines with the striker spring and inertial reverse became the mostly used in practice (Fig. 2).
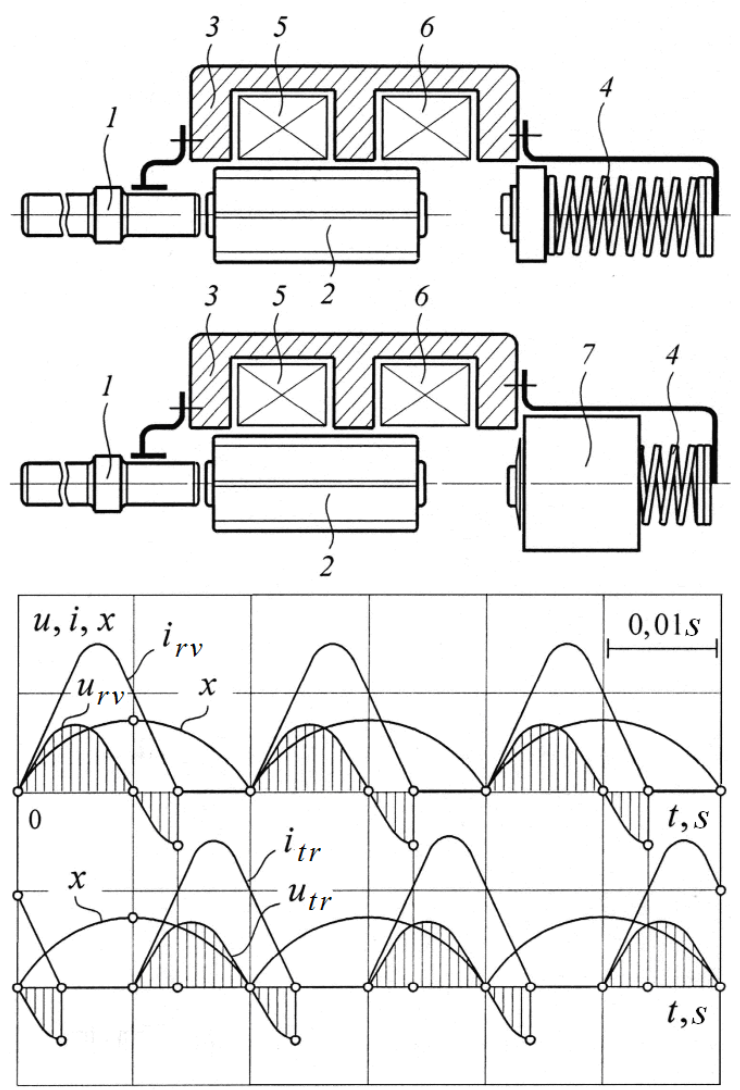

Fig. 2. Synchronous electromagnetic machine with the striker moved by constant electromagnetic forces

Such schemes were applied in impact units of the electric tools SC-2, IE-4207, IE-4709, IE-4724 and ERP-1000. The operating cycle is implemented by the two-winding system accelerating the impact mass by electromagnetic forces in travel and reverse directions.

The total impact unit operating cycle corresponds to one voltage period, providing synchronous impact frequency $n_{\mathrm{im}}=50 \mathrm{~s}^{-1} \quad\left(3000 \mathrm{~min}^{-1}\right)$ and operating cycle duration $t_{\mathrm{c}}=0,02 \mathrm{~s}$.

The striker reciprocal motion is synchronized by voltage half-waves of alternate polarities generated by the half-wave rectifier at constant electromagnetic forces acting on the striker.

Impact frequency and current can be reduced in the synchronous machine with a free running-out of the striker in the travel winding (Fig. 3). It is also possible if a free runningout of the striker takes place in the reverse winding (Fig. 4).
The striker gets necessary kinetic energy from three current pulses during one total operating cycle.
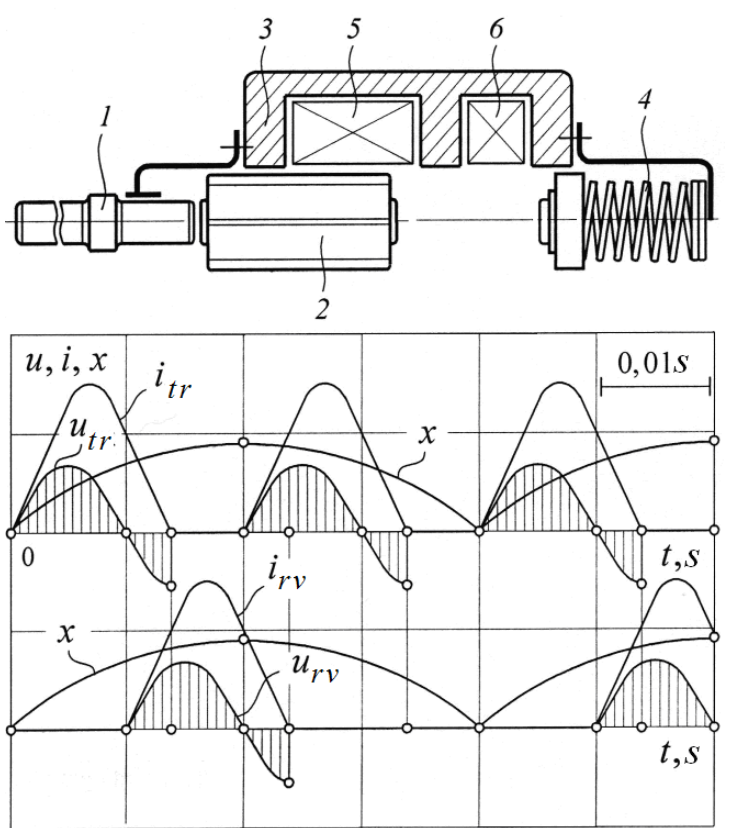

Fig. 3. Synchronous electromagnetic machine with a free running-out of the striker in the travel winding

Changing polarity sequence of voltage half-waves applied to the travel and reverse windings can transform the scheme in Fig. 3 to the scheme in Fig. 4.

If the power source frequency is $50 \mathrm{~Hz}$, then the total operating cycle corresponds to two voltage periods, providing synchronous impact frequency $n_{\mathrm{im}}=25 \mathrm{~s}^{-1}\left(1500 \mathrm{~min}^{-1}\right)$ and operating cycle duration $t_{\mathrm{c}}=0,04 \mathrm{~s}$. In the scheme in Fig. 5 the striker kinetic energy is accumulated from three current pulses during one operating cycle.

To increase machine impact power the control method has been proposed. The essence of this method is in applying the first two voltage half-waves of opposite polarities to the reverse winding and the third half-wave to the travel winding. Such half-wave sequence is periodic one with no pause between cycles (Fig. 5).

The scheme in Fig. 5 is not similar to the schemes in Fig. 3 and Fig. 4. In this variant synchronous impact frequency is $n_{\mathrm{im}}=33,3 \mathrm{~s}^{-1}\left(2000 \mathrm{~min}^{-1}\right)$ and operating cycle duration is $t_{\mathrm{c}}=0,03 \mathrm{~s}$.

Fig. 6 shows the operating cycle with a free running-out of the striker in the travel and reverse winding. The free runningout is implemented by the pause between half-waves applied to the travel and reverse winding when the striker is moving mechanically towards the spring buffer or the tool [15]. In the proposed control method both windings are use twice per one operating cycle, and the striker accumulates necessary kinetic energy from current pulses. 

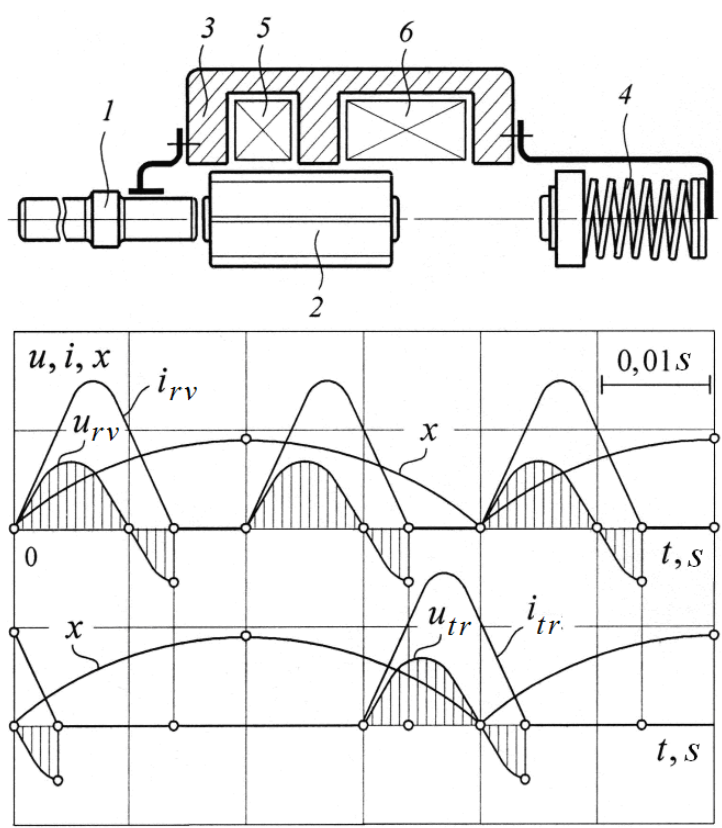

Fig. 4. Synchronous electromagnetic machine with a free running-out of the striker in the reverse winding
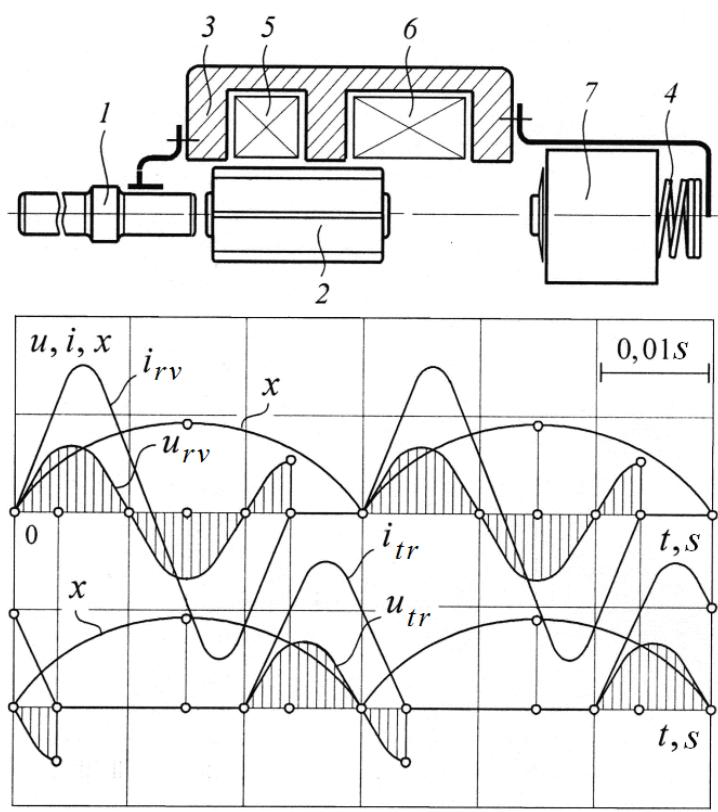

Fig. 5. Synchronous electromagnetic machine with the striker inertial reverse and reduced impact frequency

The total operating cycle of the impact unit is performed during three voltage periods, providing synchronous impact frequency $16,7 \mathrm{~s}^{-1}\left(1000 \mathrm{~min}^{-1}\right)$ and operating cycle duration $t_{\mathrm{c}}=0,06 \mathrm{~s}$.

If an impact is partially elastic, only some part of striker kinetic energy is transferred to the tool and then to the deformed medium. Other part of this energy returns back to the mechanical vibratory system during the striker idle mode, and it is partially spent on deformation and heating, leading to kinetic energy loss.

The amount of energy transferred to the deformed medium and the amount of energy returned back to the mechanical system depend on medium properties, impact system parameters and parameters of the force impulse acting on the medium.
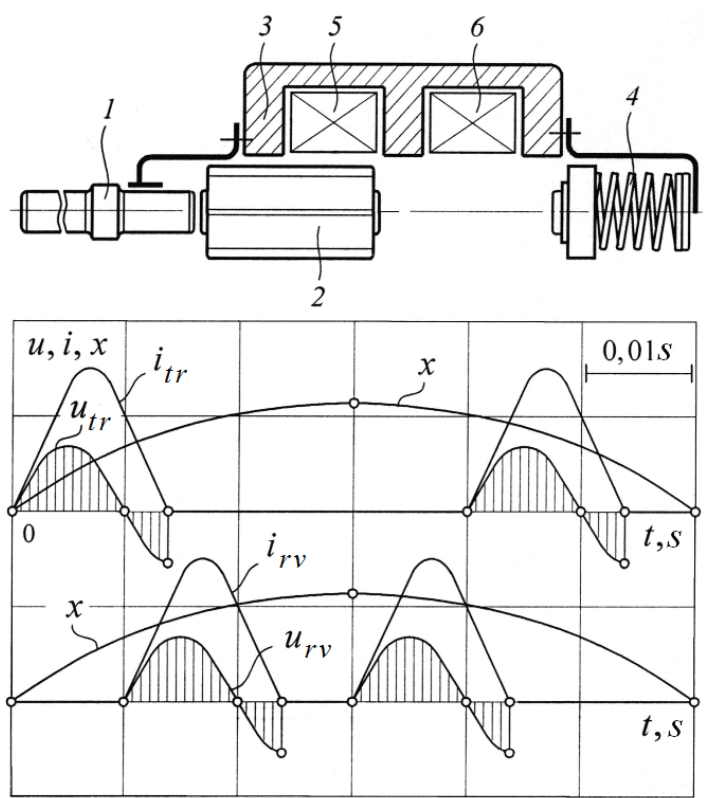

Fig. 6. Synchronous electromagnetic machine with a free running-out of the the striker in the travel and reverse windings

When an electromagnetic vibratory impact system is simulated, it is important to take into account force impulses interaction if the impact is partially elastic and kinetic energy loss takes place.

\section{THEORY}

The design diagram in Fig. 7 allows one to understand the impact interaction process between the striker and deformed medium and helps to estimate the influence of different parameters of the electromechanical vibratory impact system on the energy transfer.
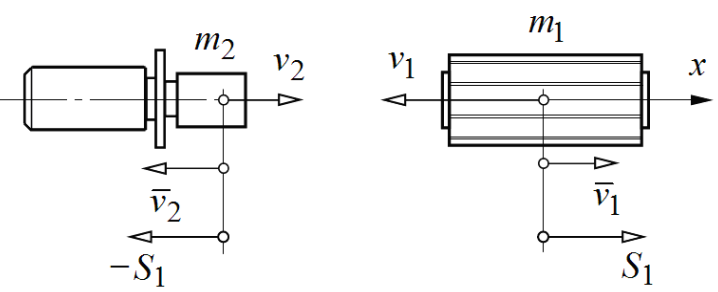

Fig.7. Design diagram of the impact interaction between the striker and the tool 
The direct central impact of two bodies consists of two steps: before the impact and after it. Such process division allows deriving striker and tool mass centers velocities after the partially elastic impact:

$$
\left\{\begin{array}{l}
\bar{v}_{1}=\bar{v}+k_{\mathrm{r}}\left(\bar{v}-\bar{v}_{1}\right), \\
\bar{v}_{2}=\bar{v}+k_{\mathrm{r}}\left(\bar{v}-\bar{v}_{2}\right),
\end{array}\right.
$$

where $v_{1}, v_{2}$ are correspondingly the striker and tool mass centers velocities before the impact, $\bar{v}_{1}, \bar{v}_{2}$ are correspondingly the striker and tool mass centers velocities after the impact, $\bar{v}$ is the velocity of the mass center if the impact is perfectly inelastic, $k_{\mathrm{r}}$ is the velocity recovery factor.

With respect to the momentum conservation law the mass center velocity for the perfectly inelastic impact is:

$$
\bar{v}=\frac{m_{1} v_{1}+m_{2} v_{2}}{m_{1}+m_{2}}
$$

If it is assumed that the tool velocity $v_{2} \approx 0$ at the impact time moment, then with respect to (1) and (2), the striker and tool mass center velocities after the impact are:

$$
\left\{\begin{array}{l}
\bar{v}_{1}=v_{1} \frac{m_{2}}{m_{1}+m_{2}}\left(\frac{m_{1}}{m_{2}}-k_{\mathrm{r}}\right), \\
\bar{v}_{2}=v_{1} \frac{m_{1}}{m_{1}+m_{2}}\left(1+k_{\mathrm{r}}\right)
\end{array} .\right.
$$

The impact causes the striker reverse. Hence the striker bouncing from the tool has the velocity $\bar{v}_{1} \leq 0$.

The striker bouncing factor is a function of impact system parameters with respect to the velocity sign:

$$
k_{\mathrm{b}}=\frac{-\bar{v}_{1}}{v_{1}}=\frac{m_{2}}{m_{1}+m_{2}}\left(k_{\mathrm{r}}-\frac{m_{1}}{m_{2}}\right) \text {. }
$$

The function $k_{\mathrm{b}}=f\left(\frac{m_{1}}{m_{2}}, k_{\mathrm{r}}\right)$ in Fig. 8 describes the impact system parameters influence on deformation with respect to kinetic energy loss. The plot in Fig. 8 shows the striker velocity after the impact (bouncing velocity) relative to the striker velocity before the impact.

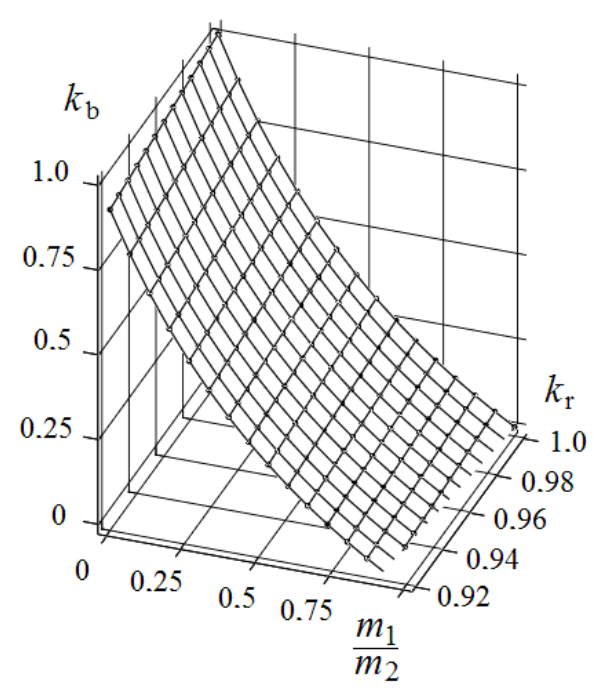

Fig. 8. Bouncing factor function $k_{\mathrm{b}}=f\left(\frac{m_{1}}{m_{2}}, k_{\mathrm{r}}\right)$

When the striker is bouncing, the kinetic energy returned to the mechanical system is:

$$
T_{1}=\frac{m_{1} \bar{v}_{1}^{2}}{2}=\frac{m_{1} v_{1}^{2}}{2}\left[\frac{m_{2}}{m_{1}+m_{2}}\left(k_{\mathrm{r}}-\frac{m_{1}}{m_{2}}\right)\right]^{2}
$$

The effective work produced by an electromagnetic motor is impulsive. If the recovery factor is constant, it can be estimated through the striker kinetic energy transferred to the tool during the impact:

$$
T_{2}=\frac{m_{2} \bar{v}_{2}^{2}}{2}=\frac{m_{2} v_{1}^{2}}{2}\left[\frac{m_{1}}{m_{1}+m_{2}}\left(1+k_{\mathrm{r}}\right)\right]^{2} .
$$

The kinetic energy loss during the impact is:

$$
\Delta T=T-T_{1}-T_{2}=\frac{m_{1} v_{1}^{2}}{2}\left[1-k_{\mathrm{b}}^{2}-\frac{m_{1} m_{2}}{\left(m_{1}+m_{2}\right)^{2}}\left(1+k_{\mathrm{r}}\right)^{2}\right],
$$

where $T_{1}=\frac{m_{1} v_{1}^{2}}{2}$ is the striker kinetic energy before the impact.

The impact energy transfer efficiency factor with respect to kinetic energy loss on deformation is:

$$
k_{\mathrm{e}}=\frac{T-T_{1}}{T}=1-\left[\frac{m_{2}}{m_{1}+m_{2}}\left(k_{\mathrm{r}}-\frac{m_{1}}{m_{2}}\right)\right]^{2} .
$$


The function $k_{\mathrm{e}}=f\left(\frac{m_{1}}{m_{2}}, k_{\mathrm{r}}\right)$, describing the amount of energy transferred to the deformed medium relative to the total striker kinetic energy over the operating cycle is stated in Fig. 9.

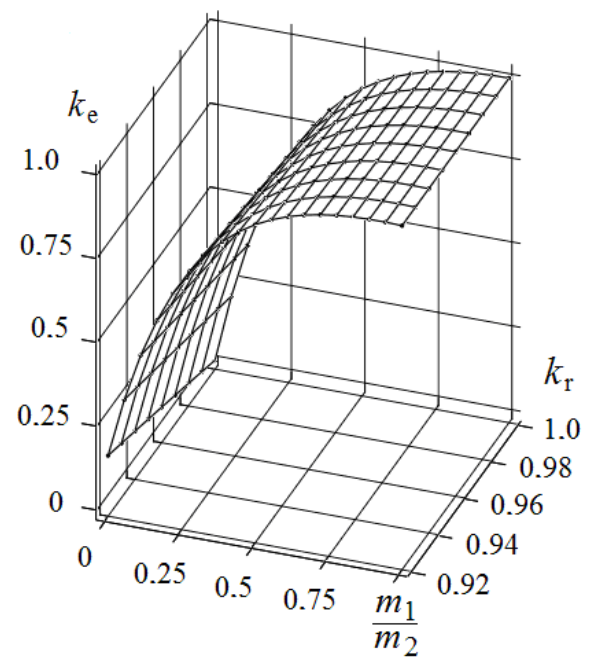

Fig. 9. The efficiency factor $k_{\mathrm{e}}=f\left(\frac{m_{1}}{m_{2}}, k_{\mathrm{r}}\right)$

\section{RESULTS AND CONCLUSION}

Simplifying interrelations between parameters of the electromechanical impact system limits capabilities of the exact dynamic analysis and synthesis of such systems operating only in transient modes with kinetic energy loss.

The dependences reflecting the influence of the parameters of the impact system with kinetic energy loss on deformation and incomplete recovery of volumes of impact masses have been established for the impact energy exchange accompanied by kinetic energy transfer to the deformed medium.

\section{References}

[1] V.P. Pevchev, "Principal dimensions of the short-stroke electromagnetic motor for a seismic wave generator" Journal of Mining Science, vol. 45, Num. 4, pp. 372-381, 2009.

[2] K.M Usanov, G.G Ugarov, V.I. Moshkin, Linear impulse electromagnetic drive of machines with self-contained power supplies, Kurgan: Kurgan University, p. 284, 2006.
[3] Y.N. Zhuravlyov, S.G. Matcevich, F.G. Kochevin, "Low-frequency electrodynamic vibrator with magnetically suspended movable part", Proc. of the 4th Intern. Conf. on Motion and Vibration Control, vol. 3, pp. $1063-1067,1998$

[4] K.M. Usanov, A.V. Volgin, E.A. Chetverikov, V.A. Kargin, A.P. Moiseev, Z.I. Ivanova, "Power electromagnetic strike machine for engineering-geological surveys", IOP Conference Series: Earth and Environmental Science [Electronic resource], p. 032049, 2017.

[5] G.G Ugarov, V.Yu. Neiman, "Evaluation of operating conditions for electromagnetic impactors", Journal of Mining Science, vol. 32, Num. 4, pp. 305-312, 1996.

[6] L.A. Neiman, V.Y. Neiman, "Linear synchronous electromagnetic machines for low-frequency impact technologies", Russian Electrical Engineering, vol. 85, Num. 12, pp. 752-756, 2014.

[7] V.K. Kadykov, N.V. Russova, G.P. Svintsov, A.V. Sizov, "The generalized experimental dependences of flux distribution, interlinkage, and magnetomotive force in direct current valve electromagnetic systems with circular pole terminals", Russian Electrical Engineering. vol. 78, Num. 4, pp. 207-212, 2007.

[8] V.I. Moshkin, D.N. Shestakov, S.Y. Pomyalov, G.G. Ugarov, "Mathematical simulation of electromagnetic pulse linear motors", Conference Proceedings, International Conference on Actual Problems of Electron Devices Engineering, APEDE 2014, pp. 348-352, 2014.

[9] L.A. Neyman, V.Y. Neyman, A.S. Shabanov, "Vibration dynamics of an electromagnetic drive with a half-period rectifier", The 18 international conference of young specialists on micro/nanotechnologies and electron devices, EDM 2017: proc., Altai, Erlagol, 29 June - 3 July 2017, pp. 503-506, 2017.

[10] R.R. Sattarov, "Electromechanical transients in passive suspension systems with eddy current dampers", 2016 9th International Conference on Power Drives Systems, ICPDS 2016, Conference Proceedings 9, p. 7756676, 2016.

[11] B.F. Simonov, V.Y. Neyman, A.S. Shabanov, "New conception of an electromagnetic drive for a vibration source in hole", The 18 international conference of young specialists on micro/nanotechnologies and electron devices, EDM 2017: proc., Altai, Erlagol, 29 June - 3 July 2017, pp. 507-510, 2017.

[12] Y.I. Klimenko, D.V. Batishchev, A.V. Pavlenko, V.P. Grinchenkov, "Design of a linear electromechanical actuator with an active vehicle suspension system", Russian Electrical Engineering, vol. 86, Num. 10, pp. 588-593, 2015.

[13] A.V. Pavlenko, A.A. Gummel', D.V. Batishchev, E. Baumbach, “A control algorithm for the magnetic drive of an air-pulse valve of an internal combustion engine", Russian Electrical Engineering, vol. 87, Num. 4, pp. 189-193, 2016.

[14] V.Y. Neiman, "Integrated linear electromagnetic motors for pulsed technologies", Russian Electrical Engineering, vol. 74, Num. 9, pp. 3035, 2003.

[15] D.V. Batischev, A.V. Pavlenko, "On designing electromagnetic drives operating under conditions of high vibrations", Russian Electrical Engineering, vol. 83, Num. 8, pp. 423, 2012.

[16] A.A. Tatevosyan, A.S. Tatevosyan, "Calculation of magnetic system of the magnetoelectric machines", Dynamics of Systems, Mechanisms and Machines, Dynamics 2014 Proceedings, p. 7005698, 2014. 